\title{
Specific Distribution of Phosphatidylglycerol to Photosystem Complexes in the Thylakoid Membrane
}

\author{
Koichi Kobayashi*, Kaichiro Endo and Hajime Wada \\ Department of Life Sciences, Graduate School of Arts and Sciences, The University of Tokyo, Tokyo, Japan
}

The thylakoid membrane is the site of photochemical and electron transport reactions of oxygenic photosynthesis. The lipid composition of the thylakoid membrane, with two galactolipids, one sulfolipid, and one phospholipid, is highly conserved among oxygenic photosynthetic organisms. Besides providing a lipid bilayer matrix, thylakoid lipids are integrated in photosynthetic complexes particularly in photosystems I and $\|$ and play important roles in electron transport processes. Thylakoid lipids are differentially allocated to photosynthetic complexes and the lipid bilayer fraction, but

OPEN ACCESS

Edited by:

Rebecca L. Roston, University of Nebraska-Lincoln,

United States

Reviewed by:

Conrad Mullineaux,

Queen Mary University of London, United Kingdom

Julian Eaton-Rye,

University of Otago, New Zealand

*Correspondence:

Koichi Kobayash

kkobayashi@bio.c.u-tokyo.ac.jp

Specialty section: This article was submitted to Plant Cell Biology,

a section of the journal

Frontiers in Plant Science

Received: 27 September 2017 Accepted: 06 November 2017

Published: 20 November 2017

Citation:

Kobayashi K, Endo K and Wada H (2017) Specific Distribution

of Phosphatidylglycerol to Photosystem Complexes in the Thylakoid Membrane.

Front. Plant Sci. 8:1991. doi: 10.3389/fpls.2017.01991 distribution of each lipid in the thylakoid membrane is unclear. In this study, based on published crystallographic and biochemical data, we estimated the proportions of photosynthetic complex-associated and bilayer-associated lipids in thylakoid membranes of cyanobacteria and plants. The data suggest that $\sim 30 \mathrm{~mol} \%$ of phosphatidylglycerol (PG), the only major phospholipid in thylakoid membranes, is allocated to photosystem complexes, whereas glycolipids are mostly distributed to the lipid bilayer fraction and constitute the membrane lipid matrix. Because PG is essential for the structure and function of both photosystems, PG buried in these complexes might have been selectively conserved among oxygenic phototrophs. The specific and substantial allocation of PG to the deep sites of photosystems may need a unique mechanism to incorporate PG into the complexes possibly in coordination with the synthesis of photosynthetic proteins and pigments. Keywords: thylakoid membrane, monogalactosyldiacylglycerol, digalactosyldiacylglycerol,
sulfoquinovosyldiacylglycerol, phosphatidylglycerol, lipid bilayer, photosystem I, photosystem II

\section{INTRODUCTION}

The thylakoid membrane is the site of photochemical and electron transport reactions in oxygenic phototrophs. In thylakoid membranes, glycerolipids form a bilayer matrix, in which photosynthetic protein-cofactor complexes such as photosystem I (PSI), photosystem II (PSII), cytochrome (Cyt) $b_{6} / f$ complex and ATP synthase are embedded. The hydrophobic lipid bilayer prevents free diffusion of ions and allows for creating a proton gradient while enabling lateral diffusion of the photosynthetic complexes and the mobile electron carriers for efficient electron transport reactions.

The lipid composition of the thylakoid membrane is highly conserved among oxygenic phototrophs (Murata et al., 1981; Mendiola-Morgenthaler et al., 1985; Dorne et al., 1990). 
Galactolipids, monogalactosyldiacylglycerol (MGDG) and digalactosyldiacylglycerol (DGDG), account for $\sim 50$ and $\sim 30 \mathrm{~mol} \%$ of total thylakoid lipids, respectively. Thylakoids contain another unique glycolipid, sulfoquinovosyldiacylglycerol (SQDG), which constitutes $\sim 10 \%$ of the total lipids. The remaining $\sim 10 \%$ is phosphatidylglycerol (PG), the only major phospholipid in thylakoids.

Each lipid class has a different physicochemical property depending on the nature of its head group. MGDG and DGDG are neutral lipids with uncharged polar head groups. MGDG has a cone-like shape with a small galactose head group and flexible poly-unsaturated fatty acid tails and thus tends to form a non-bilayer lipid phase in mixture with water (Shipley et al., 1973; Jouhet, 2013). By contrast, DGDG has a more bulky head group with two galactoses, which gives DGDG a cylindrical shape and allows for forming a bilayer lamellar phase. The MGDG/DGDG ratio strongly affects the phase behavior of the lipid bilayer and may be important for the structure and stability of the thylakoid membrane (Murphy, 1982; Demé et al., 2014). SQDG and PG, which are both bilayer lipids, have negative charge at neutral $\mathrm{pH}$ and are classified as acidic lipids. PG and SQDG are in part functionally redundant in both cyanobacteria and plant chloroplasts, as we describe later, presumably because of their common acidic properties in polar head groups.

Besides providing a lipid bilayer matrix, thylakoid lipids are integrated in PSII (Loll et al., 2005, 2007; Sakurai et al., 2006; Umena et al., 2011; Wei et al., 2016), Cyt $b_{6} / f$ (Kurisu et al., 2003; Stroebel et al., 2003; Baniulis et al., 2009; Hasan et al., 2011), PSI (Jordan et al., 2001; Kubota et al., 2010; Qin et al., 2015; Mazor et al., 2017), and light-harvesting complexes (LHCs) (Liu et al., 2004; Standfuss et al., 2005; Wei et al., 2016) and play important roles in electron transport processes (Kobayashi et al., 2016b). Because these photosynthetic complexes account for large quantities of the thylakoid membrane, substantial amounts of lipids would be allocated to the complexes as structural components. Based on published crystallographic and biochemical data, we estimated the amount of photosynthetic complex-associated lipids and lipid composition in the thylakoid lipid bilayer. The results indicated a preferential distribution of PG to photosystems and a minor contribution to the thylakoid lipid bilayer in plant chloroplasts and cyanobacteria.

\section{DISTRIBUTION OF LIPIDS IN THE THYLAKOID MEMBRANE IN CYANOBACTERIA}

Jordan et al. (2001) first revealed the crystal structure of PSI from Thermosynechococcus elongatus at 2.5- $\AA$ resolution. In the PSI trimer, 1 MGDG and 3 PG molecules per monomer were identified. The same lipid configuration was observed in the PSI crystal structure of Synechocystis sp. PCC 6803 (Mazor et al., 2014). Meanwhile, crystallographic analysis of the PSII dimer from Thermosynechococcus vulcanus, the species closely related to $T$. elongatus, at $1.9-\AA$ resolution identified 20 lipid molecules (6 MGDGs, 5 DGDGs, 4 SQDGs, and 5 PGs) and 3 unknown diglycerides per monomer (Umena et al., 2011). A similar lipid composition was revealed by lipid analysis of PSII dimers purified from T. vulcanus and Synechocystis sp. PCC 6803 (Sakurai et al., 2006), which supports the validity of these results.

From the data in Sakurai et al. (2006), we computed the molar ratio of each lipid to chlorophyll (Chl) in the thylakoid fraction from T. vulcanus (Table 1). Because of no available data for the total PSI and PSII content in Thermosynechococcus species, in this study, we used the data for PSI $(7.5 \mathrm{mmol} / \mathrm{mol} \mathrm{Chl})$ and PSII $(3.2 \mathrm{mmol} / \mathrm{mol} \mathrm{Chl})$ content in another thermophilic cyanobacterium Synechococcus lividus (Melis and Brown, 1980) for a rough estimation. Similar content in Synechocystis sp. PCC 6714 (Fujita and Murakami, 1987; Murakami et al., 1997) supports the validity of this assumption. In combination with the crystallographic data (Jordan et al., 2001; Umena et al., 2011), these biochemical data allowed us to estimate 1.3 and $2.8 \mathrm{~mol} \%$ of total thylakoid lipids allocated to PSI and PSII, respectively (Figure 1A). Of note, $\sim 30 \%$ of total PG molecules is associated with photosystems, whereas only a small portion $(\sim 3 \%)$ is allocated to photosystems for other lipid classes. As a result, PG accounts for only $4.6 \mathrm{~mol} \%$ of total lipids outside photosystems, presumably mainly in the lipid bilayer fraction. These data demonstrate that PG, the only phospholipid in cyanobacteria, is preferentially allocated to photosystem complexes, whereas glycolipids predominantly constitute the lipid bilayer of the thylakoid membrane. We should note that because the PSI-to-PSII ratios in cyanobacteria flexibly change from $\sim 0.5$ to $\sim 5$ depending on growth conditions, particularly light quality and quantity (Fujita and Murakami, 1987; Samson et al., 1994; Murakami et al., 1997), the proportion of PG in photosystems may also range from $\sim 45$ to $\sim 23 \mathrm{~mol} \%$ of total thylakoid PG.

\section{DISTRIBUTION OF LIPIDS IN THE THYLAKOID MEMBRANE IN PLANTS}

Plants contain LHC antenna complexes in addition to core complexes in both PSI and PSII complexes. The crystal structure of the PSI-LHCI complex from pea at 2.8- $\AA$ resolution includes 10 lipid molecules (3 MGDGs, 1 DGDG, and 6 PGs) per monomer (Qin et al., 2015). Similar to T. elongatus PSI (Jordan et al., 2001), 1 MGDG, 1 DGDG, and 3 PG molecules were associated with the PSI core in pea. The more recent analysis of the pea PSI-LHCI complex at 2.6- $\AA$ resolution identified 27 lipid molecules (19 MGDGs, 4 DGDGs, and 4 PGs), mostly located between PSI and LHCI, in addition to the five lipids in the core region (Mazor et al., 2017).

Single-particle cryo-electron microscopic analysis of the $\mathrm{C}_{2} \mathrm{~S}_{2}$-type PSII-LHCII supercomplex (C; PSII core complex, $S$; strongly associated LHCII trimer) from spinach at 3.2resolution revealed 21 lipid molecules per monomeric complex (Wei et al., 2016). The lipid content (5 MGDGs, 4 DGDGs, 3 SQDGs, and 4 PGs) in the spinach PSII core is similar to that in T. vulcanus (Umena et al., 2011). In addition, 
TABLE 1 | Lipid content in the thylakoid membrane and photosystem complexes.

\begin{tabular}{|c|c|c|c|c|c|c|c|}
\hline & & & & Thylakoid & PSI & PSII & Other \\
\hline \multirow[t]{7}{*}{ Cyanobacteria } & & PSI & & 7.52 & - & - & - \\
\hline & & PSII & & 3.24 & - & - & - \\
\hline & Lipids & & Total & 2350 & $30.1(4)$ & $64.8(20)$ & 2230 \\
\hline & & & MGDG & 1020 & $7.52(1)$ & $19.4(6)$ & 995 \\
\hline & & & DGDG & 602 & $0(0)$ & $16.2(5)$ & 586 \\
\hline & & & SQDG & 583 & $0(0)$ & $13.0(4)$ & 570 \\
\hline & & & PG & 143 & $22.6(3)$ & $16.2(5)$ & 104 \\
\hline \multirow[t]{7}{*}{ Plants } & & PSI & & 2.25 & - & - & - \\
\hline & & PSII & & 2.99 & - & - & - \\
\hline & Lipids & & Total & 1950 & $72.0(32)$ & $89.7(30)$ & 1790 \\
\hline & & & MGDG & 840 & $45.0(20)$ & $15.0(5)$ & 780 \\
\hline & & & DGDG & 510 & $11.3(5)$ & $12.0(4)$ & 487 \\
\hline & & & SQDG & 380 & $0(0)$ & $9.00(3)$ & 371 \\
\hline & & & $P G$ & 220 & $15.8(7)$ & $53.8(18)$ & 150 \\
\hline
\end{tabular}

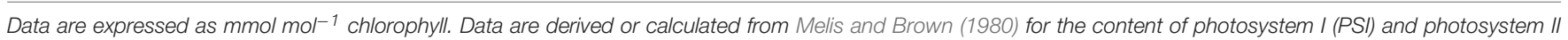

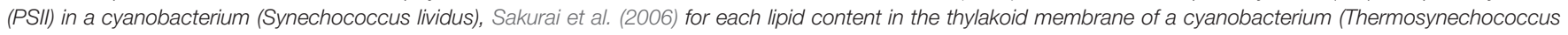

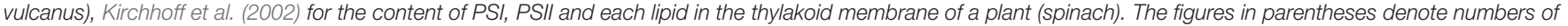

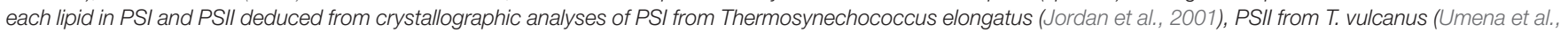
2011), PSI-light harvesting complex (LHC) I from pea (Mazor et al., 2017), and PSII-LHC/l from spinach (Wei et al., 2016).

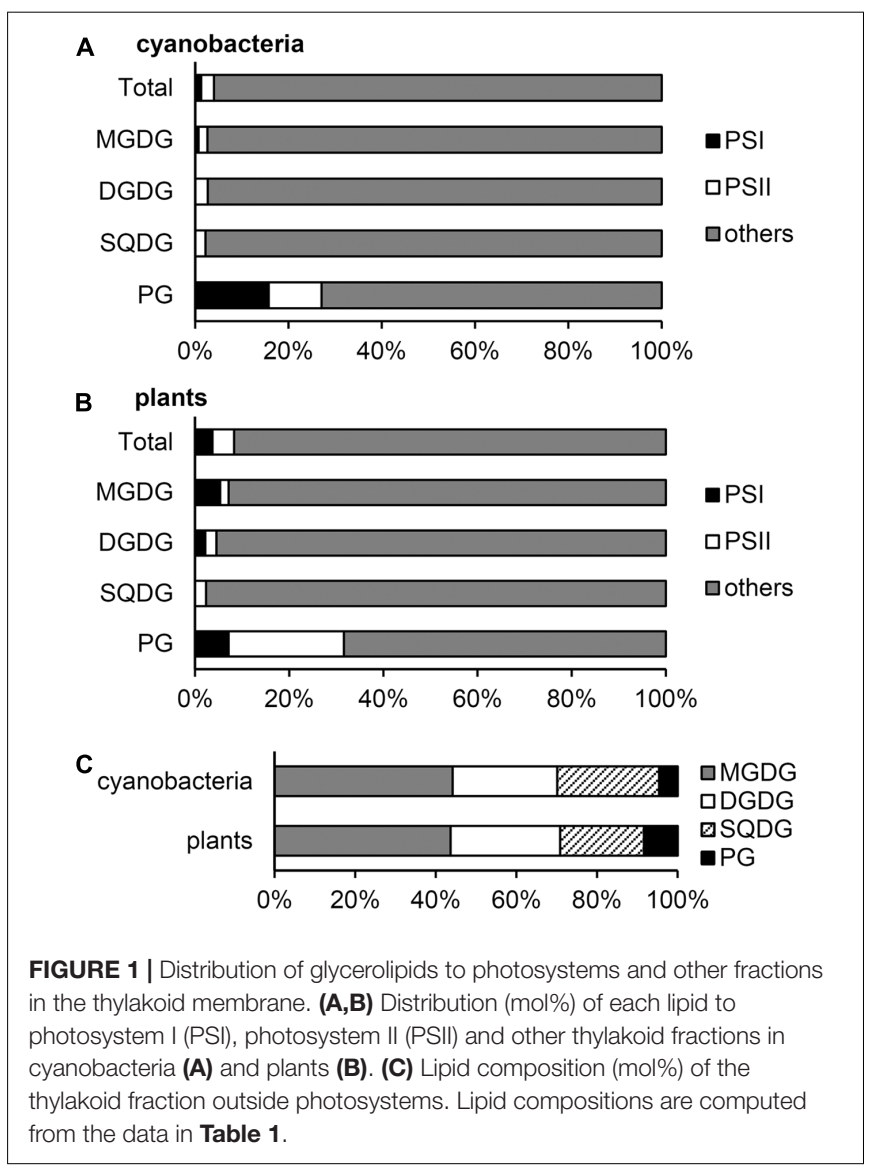

5 PG molecules were associated with the LHCII complex composed of 1 trimeric LHCII with 3 PGs, 1 monomeric CP26 with 1 PG, and 1 monomeric CP29 with 1 PG. X-ray crystallography analyses of the LHCII trimer from spinach at $2.7 \AA$ (Liu et al., 2004) and from pea at $2.5 \AA$ (Standfuss et al., 2005) both identified one PG molecule per monomer, which is consistent with the data in the $\mathrm{C}_{2} \mathrm{~S}_{2}$-type PSIILHCII supercomplex (Wei et al., 2016). By contrast, 2 DGDG molecules per monomer observed at the periphery of the crystallized LHCII trimers (Liu et al., 2004; Standfuss et al., 2005) were missing in the $\mathrm{C}_{2} \mathrm{~S}_{2}$-type supercomplex. Because binding sites of DGDG differed between spinach and pea data, these DGDGs may be integrated into the LHCII trimer during crystallization processes. Besides the $\mathrm{C}_{2} \mathrm{~S}_{2}$-type complex, other forms of the PSII-LHCII supercomplex such as $\mathrm{C}_{2} \mathrm{~S}_{2} \mathrm{M}_{2}$ type (M; moderately associated LHCII trimer) have been identified in plant thylakoids (Pan et al., 2013). Biochemical analysis revealed that a single PSII-LHCII unit contains 250 Chls on average (Ghirardi et al., 1986), which would correspond to 4 major LHCII trimers (12 subunits in total) and 3 minor monomeric LHCIIs, presumably CP24, CP26, and CP29, per PSII monomer (Peter and Thornber, 1991; Pan et al., 2013). This estimation is consistent with the 3.8-times higher amount of the LHCII trimer than the PSII monomer on a molar basis in spinach thylakoids (Kirchhoff et al., 2002). From these data, we assumed that PSII-LHCII complexes contain a total of 30 lipids (5 MGDGs, 4 DGDGs, 3 SQDGs, and 18 PGs) per PSII monomer, on average.

Although the relative abundance of PSI, PSII, and LHCII in plants greatly differs according to growth conditions, in this study, we estimated lipid content in photosystem complexes based on the data in spinach thylakoids (Table 1) (Kirchhoff et al., 2002). The estimation revealed that PSI-LHCI and PSIILHCII contain 3.7 and $4.6 \mathrm{~mol} \%$, respectively, total lipids in the thylakoid membrane (Figure 1B). As in cyanobacteria, $\sim 30 \mathrm{~mol} \%$ of total PG in the thylakoid membrane is allocated to photosystems in plant chloroplasts, and thus the contribution of 
PG to other lipid fractions $(8.4 \mathrm{~mol} \%)$ is lower than that of SQDG (20.7 mol\%) (Figure 1C).

\section{DISTRIBUTION OF LIPIDS IN OTHER THYLAKOID FRACTIONS}

The Cyt $b_{6} / f$ complex mediates electron transfer from PSII to PSI and generates an electrochemical proton gradient across the thylakoid membrane together with water splitting in PSII. The crystal structure of the dimeric Cyt $b_{6} / f$ complex has been determined for Chlamydomonas reinhardtii (Stroebel et al., 2003), Mastigocladus laminosus (Kurisu et al., 2003) and Nostoc sp. PCC 7120 (Baniulis et al., 2009). From these data, one SQDG molecule was identified with two synthetic phosphatidylcholine and four detergents per monomer. In addition, mass spectroscopy analysis of spinach Cyt $b_{6} / f$ detected MGDG, DGDG, and PG along with SQDG (Hasan et al., 2011). Although two neutral lipids at the lumenal side were modeled as MGDGs in the C. reinhardtii Cyt $b_{6} / f$ (Stroebel et al., 2003), one may be a DGDG molecule based on the mass spectroscopy data. Furthermore, the presence of stoichiometric amounts of PG and SQDG in spinach Cyt $b_{6} / f$ suggests an equal amount of these anionic lipids in the structure (Hasan et al., 2011). Considering the content of Cyt $b_{6} / f$ of $\sim 1.0 \mathrm{mmol} / \mathrm{mol} \mathrm{Chl} \mathrm{in}$ plants (Kirchhoff et al., 2002; Schöttler and Toth, 2014) and $\sim 5.0 \mathrm{mmol} / \mathrm{mol} \mathrm{Chl} \mathrm{in} \mathrm{cyanobacteria} \mathrm{(Fujita} \mathrm{and} \mathrm{Murakami,}$ 1987), we roughly estimated that $0.3 \sim 0.9 \mathrm{~mol} \%$ of total thylakoid lipids are integrated in the Cyt $b_{6} / f$ complexes with stoichiometry of 1 MGDG, 1 DGDG, 1 SQDG, and 1 PG per monomer.

For ATP synthesis, ATP synthase uses a proton gradient across the thylakoid membrane. In animals, cardiolipin (diphosphatidylglycerol), the anionic lipid produced from PG and CDP-diacylglycerol, is an essential component of mitochondrial ATP synthase (Santiago et al., 1973; Laird et al., 1986). Computer simulations demonstrated that cardiolipin specifically and transiently interacts with the rotating c-ring of yeast ATP synthase (Duncan et al., 2016). In the thylakoid membrane, containing no cardiolipin, the anionic lipid PG or SQDG may be specifically associated with ATP synthase, although the lipid content in the thylakoid ATP synthase remains undetermined.

Considering the predominant amount of photosynthetic electron transport complexes in the thylakoid membrane (Yu et al., 1994; Herranen et al., 2004), lipids that do not integrate in these major complexes would be mostly allocated to the thylakoid lipid bilayer. We estimated that $92-95 \mathrm{~mol} \%$ of the thylakoid lipid bilayer is composed of glycolipids (Figure 1C).

\section{ROLE OF PG IN PHOTOSYSTEMS}

In general, the primary role of membrane glycerolipids is to construct the lipid bilayer. In fact, most glycolipids in thylakoid are distributed to the lipid bilayer fraction as building blocks (Figure 1). However, our estimation revealed that $\sim 30 \mathrm{~mol} \%$ of PG in the thylakoid membrane is allocated to photosystem complexes as a structural component in both cyanobacteria and plant chloroplasts, and the quantitative contribution of PG to the thylakoid lipid bilayer is low (5-8 mol\%). Because loss of PG severely impairs photosynthetic electron transport in all oxygenic phototrophs examined to date (Kobayashi et al., 2016b), this lipid would specifically serve as an essential component of photosystems.

In the crystal structure of T. vulcanus PSII, all 5 PG molecules are deeply buried near the reaction center (Umena et al., 2011). Short-term depletion (3-7 days) of PG in Synechocystis sp. PCC 6803 cells caused loss of $\sim 3$ PG molecules from PSII, accompanied by impaired electron transport from the primary $\left(Q_{\mathrm{A}}\right)$ to the secondary plastoquinone $\left(Q_{\mathrm{B}}\right)$ and destabilized oxygen-evolving complex (Sakurai et al., 2003, 2007). Moreover, site-directed mutations of PG-associated amino-acid residues of the D1 protein, which caused loss of about 1 PG molecule from the PSII complex, decreased electron transport activity and disordered PSII structures (Endo et al., 2015). These data demonstrate the essential roles of PSII-associated PG in structures and functions of the complex.

In contrast to the rapid inhibition of PSII by PG deficiency, PSI is affected only after a longer period ( $>2$ weeks) of PG depletion (Domonkos et al., 2004), which may reflect more stable association of PG with PSI. Three PG molecules are integrated in the crystal structure of T. elongatus PSI (Jordan et al., 2001). One is buried near the reaction center, and the other two locate at the periphery. Decreased PSI activity and increased monomeric PSI complexes after long-term PG deficiency suggest an important role of PG in the activity and structural organization of PSI (Domonkos et al., 2004).

In plants, PG molecules are associated with the LHCI and LHCII complexes in addition to the photosystem core complexes (Liu et al., 2004; Standfuss et al., 2005; Qin et al., 2015; Wei et al., 2016; Mazor et al., 2017). Degradation of PG by phospholipase A2 treatment disassembled the LHCII trimer into the monomeric form (Nussberger et al., 1993; Kim et al., 2007). Moreover, PG deficiency in a C. reinhardtii mutant decreased trimeric LHCII level, but PG supplementation to the mutant partially recovered the phenotype (Dubertret et al., 1994). These findings indicate a crucial role of PG in structural organization of the LHCII trimer. In addition, in the pea PSI-LHCI complex, several PG molecules are located between PSI and LHCI with MGDGs and DGDGs (Mazor et al., 2017). Because lack of any of these lipids caused disorganization of PSI-LHCI complexes in Arabidopsis thaliana mutants (Ivanov et al., 2006; Kobayashi et al., 2013, 2016a), the lipid cluster observed between PSI and LHCI may specifically function to maintain the complex structure.

The preferential allocation of PG to the deep sites of both photosystems despite the low abundance of PG in the thylakoid lipid bilayer suggests a requirement of specific mechanisms to deliver PG molecules within the protein complexes. Kopečná et al. (2015) reported that loss of PG in the Synechocystis sp. PCC 6803 mutant strongly impaired synthesis of PSI proteins and Chls. The authors proposed that a PG-containing membrane microdomain may be required for the synthesis of these 
components (Kopečná et al., 2015). Considering the low amount of PG in the thylakoid lipid bilayer, PG distribution may be restricted to a specific microdomain in the thylakoid membrane, where assembly of PG to protein-pigment complexes may take place along with synthesis of Chls and PSI.

\section{ROLE OF PG OUTSIDE PHOTOSYSTEMS}

Mutant analyses in A. thaliana, C. reinhardtii and several cyanobacteria revealed that loss of SQDG increased PG levels to maintain total anionic lipid content (Sato et al., 1995; Güler et al., 1996; Yu et al., 2002; Aoki et al., 2004; Endo et al., 2016). In T. elongatus, PG content was increased from 4 to $25 \mathrm{~mol} \%$ by disruption of SQDG biosynthesis (Endo et al., 2016). Because photosystem-associated SQDG is only $3.7 \mathrm{~mol} \%$ of the total SQDG level in Thermosynechococcus thylakoids (Figure 1A), PG produced in response to loss of SQDG would be mainly allocated to the lipid bilayer fraction. Although complete lack of SQDG alone has no or only weak effects in most photosynthetic organisms (Sato et al., 1995; Güler et al., 1996; Yu et al., 2002; Endo et al., 2016), a partial decrease in PG content along with lack of SQDG strongly impaired photosynthetic activity and growth (Güler et al., 1996; Yu and Benning, 2003; Endo et al., 2016). The result suggests an importance of maintained levels of total anionic lipids in thylakoids. Because SQDG is the major anionic lipid in the thylakoid lipid bilayer (Figure 1C), the main role of this lipid may be maintaining a certain level of anionic lipids in the membrane. By contrast, PG has more specific roles in photosystems. However, when SQDG content is decreased, PG content increases and would complement the role of SQDG in the lipid bilayer.

Under phosphate-limited conditions, PG content in A. thaliana decreased from $\sim 10$ to $\sim 5 \mathrm{~mol} \%$ of total membrane lipids, with SQDG content inversely increased from $\sim 6$ to $\sim 11$ mol\% (Essigmann et al., 1998). Because photosystemassociated PG is indispensable for photosynthesis, PG molecules that are not integrated in the complexes may be preferentially degraded under phosphate limitation. This assumption is supported by the fact that phosphate limitation severely affected photosynthetic electron transport in PG-deficient A. thaliana mutants but not the wild type (Kobayashi et al., 2015). Thus, increased SQDG under phosphate limitation would mainly replace PG outside photosystem complexes. A similar

\section{REFERENCES}

Aoki, M., Sato, N., Meguro, A., and Tsuzuki, M. (2004). Differing involvement of sulfoquinovosyl diacylglycerol in photosystem II in two species of unicellular cyanobacteria. Eur. J. Biochem. 271, 685-693. doi: 10.1111/j.1432-1033.2003. 03970.x

Awai, K., Watanabe, H., Benning, C., and Nishida, I. (2007). Digalactosyldiacylglycerol is required for better photosynthetic growth of Synechocystis sp. PCC6803 under phosphate limitation. Plant Cell Physiol. 48, 1517-1523. doi: 10.1093/pcp/pcm134

Baniulis, D., Yamashita, E., Whitelegge, J. P., Zatsman, A. I., Hendrich, M. P., Hasan, S. S., et al. (2009). Structure-function, stability, and chemical modification of the cyanobacterial cytochrome $b_{6} f$ complex from Nostoc sp. PCC 7120. J. Biol. Chem. 284, 9861-9869. doi: 10.1074/jbc.M809196200 relationship between PG and SQDG under phosphate limitation is observed in S. elongatus PCC 7942 (Güler et al., 1996) but not Synechocystis sp. PCC 6803 (Awai et al., 2007) or T. elongatus (Endo et al., 2016). PG content is substantially low in T. elongatus ( $\sim 4 \mathrm{~mol} \%)$ as compared with S. elongatus (16.6 mol\%) even under phosphate-sufficient conditions (Endo et al., 2016). Conversely, SQDG content is higher in T. elongatus $(\sim 18 \mathrm{~mol} \%)$ than S. elongatus (10.3 mol\%). T. elongatus may already decrease PG content to a minimal level under phosphatesufficient conditions and thus cannot further reduce PG content under limited phosphate. Use of glycolipids for the lipid bilayer reduces spending phosphate in the membrane, which would be advantageous for growth of photosynthetic organisms particularly under phosphate-limited conditions. The specific enrichment of PG in photosystems implies an indispensable role of this lipid, which presumably could not have been replaced by glycolipids during the long evolutionary processes of oxygenic photosynthetic organisms.

\section{CONCLUSION}

In both plants and cyanobacteria, one third of total PG molecules in thylakoids is specifically integrated with photosystems to fulfill its essential role in photosynthesis. By contrast, galactolipids mainly constitute the thylakoid lipid bilayer with the anionic glycolipid SQDG, which reduces the use of phosphate in the thylakoid membrane. The specific enrichment of PG in photosystems implies a particular mechanism to assemble PG into the deep sites of the complexes, which should be evaluated in future studies.

\section{AUTHOR CONTRIBUTIONS}

KK conceived the study, analyzed data, and wrote the manuscript. $\mathrm{KE}$ analyzed data and complemented the writing. HW supervised the study and complemented the writing.

\section{FUNDING}

This work was supported by the JSPS KAKENHI Grant Number 26711016.

Demé, B., Cataye, C., Block, M. A., Maréchal, E., and Jouhet, J. (2014). Contribution of galactoglycerolipids to the 3-dimensional architecture of thylakoids. FASEB J. 28, 3373-3383. doi: 10.1096/fj.13-247395

Domonkos, I., Malec, P., Sallai, A., Kovács, L., Itoh, K., Shen, G., et al. (2004). Phosphatidylglycerol is essential for oligomerization of photosystem I reaction center. Plant Physiol. 134, 1471-1478. doi: 10.1104/pp.103.037754

Dorne, A., Joyard, J., and Douce, R. (1990). Do thylakoids really contain phosphatidylcholine? Proc. Natl. Acad. Sci. U.S.A. 87, 71-74.

Dubertret, G., Mirshahi, A., Mirshahi, M., Gerard-Hirne, C., and Tremolieres, A. (1994). Evidence from in vivo manipulations of lipid composition in mutants that the $\Delta^{3}$-trans-hexadecenoic acid-containing phosphatidylglycerol is involved in the biogenesis of the light-harvesting chlorophyll $a / b$-protein complex of Chlamydomonas reinhardtii. Eur. J. Biochem. 226, 473-482. doi: 10.1111/j.1432-1033.1994.tb20072.x 
Duncan, A. L., Robinson, A. J., and Walker, J. E. (2016). Cardiolipin binds selectively but transiently to conserved lysine residues in the rotor of metazoan ATP synthases. Proc. Natl. Acad. Sci. U.S.A. 113, 8687-8692. doi: 10.1073/pnas. 1608396113

Endo, K., Kobayashi, K., and Wada, H. (2016). Sulfoquinovosyldiacylglycerol has an essential role in Thermosynechococcus elongatus BP-1 under phosphatedeficient conditions. Plant Cell Physiol. 57, 2461-2471. doi: 10.1093/pcp/ pcw159

Endo, K., Mizusawa, N., Shen, J.-R., Yamada, M., Tomo, T., Komatsu, H., et al. (2015). Site-directed mutagenesis of amino acid residues of D1 protein interacting with phosphatidylglycerol affects the function of plastoquinone $\mathrm{Q}_{\mathrm{B}}$ in photosystem II. Photosynth. Res. 126, 385-397. doi: 10.1007/s11120-0150150-9

Essigmann, B., Güler, S., Narang, R. A., Linke, D., and Benning, C. (1998). Phosphate availability affects the thylakoid lipid composition and the expression of SQD1, a gene required for sulfolipid biosynthesis in Arabidopsis thaliana. Proc. Natl. Acad. Sci. U.S.A. 95, 1950-1955.

Fujita, Y., and Murakami, A. (1987). Regulation of electron transport composition in cyanobacterial photosynthetic system: stoichiometry among photosystem I and II complexes and their light-harvesting antennae and cytochrome $b 6 / f$ complex. Plant Cell Physiol. 28, 1547-1553.

Ghirardi, M. L., McCauley, S. W., and Melis, A. (1986). Photochemical apparatus organization in the thylakoid membrane of Hordeum vulgare wild type and chlorophyll $b$-less chlorina f2 mutant. Biochim. Biophys. Acta 851, 331-339. doi: 10.1016/0005-2728(86)90069-1

Güler, S., Seeliger, A., Härtel, H., Renger, G., and Benning, C. (1996). A null mutant of Synechococcus sp. PCC7942 deficient in the sulfolipid sulfoquinovosyl diacylglycerol. J. Biol. Chem. 271, 7501-7507. doi: 10.1074/jbc.271.13. 7501

Hasan, S. S., Yamashita, E., Ryan, C. M., Whitelegge, J. P., and Cramer, W. A. (2011). Conservation of lipid functions in cytochrome bc complexes. J. Mol. Biol. 414, 145-162. doi: 10.1016/j.jmb.2011.09.023

Herranen, M., Battchikova, N., Zhang, P., Graf, A., Sirpiö, S., Paakkarinen, V., et al. (2004). Towards functional proteomics of membrane protein complexes in Synechocystis sp. PCC 6803. Plant Physiol. 134, 470-481. doi: 10.1104/pp.103. 032326

Ivanov, A. G., Hendrickson, L., Krol, M., Selstam, E., Öquist, G., Hurry, V., et al. (2006). Digalactosyl-diacylglycerol deficiency impairs the capacity for photosynthetic intersystem electron transport and state transitions in Arabidopsis thaliana due to photosystem I acceptor-side limitations. Plant Cell Physiol. 47, 1146-1157. doi: 10.1093/pcp/pcj089

Jordan, P., Fromme, P., Witt, H. T., Klukas, O., Saenger, W., and Krauss, N. (2001). Three-dimensional structure of cyanobacterial photosystem I at $2.5 \AA$ resolution. Nature 411, 909-917. doi: 10.1038/35082000

Jouhet, J. (2013). Importance of the hexagonal lipid phase in biological membrane organization. Front. Plant Sci. 4:494. doi: 10.3389/fpls.2013. 00494

Kim, E.-H., Razeghifard, R., Anderson, J. M., and Chow, W. S. (2007). Multiple sites of retardation of electron transfer in Photosystem II after hydrolysis of phosphatidylglycerol. Photosynth. Res. 93, 149-158. doi: 10.1007/s11120-0069126-0

Kirchhoff, H., Mukherjee, U., and Galla, H. J. (2002). Molecular architecture of the thylakoid membrane: lipid diffusion space for plastoquinone. Biochemistry 41, 4872-4882. doi: 10.1021/bi011650y

Kobayashi, K., Endo, K., and Wada, H. (2016a). Multiple impacts of loss of plastidic phosphatidylglycerol biosynthesis on photosynthesis during seedling growth of Arabidopsis. Front. Plant Sci. 7:336. doi: 10.3389/fpls.2016.00336

Kobayashi, K., Endo, K., and Wada, H. (2016b). "Roles of lipids in photosynthesis," in Lipids in Plant and Algae Development, eds Y. Nakamura and Y. Li-Beisson (Berlin: Springer International Publishing), 21-49. doi: 10.1007/978-3-31925979-6_2

Kobayashi, K., Fujii, S., Sato, M., Toyooka, K., and Wada, H. (2015). Specific role of phosphatidylglycerol and functional overlaps with other thylakoid lipids in Arabidopsis chloroplast biogenesis. Plant Cell Rep. 34, 631-642. doi: 10.1007/ s00299-014-1719-z

Kobayashi, K., Narise, T., Sonoike, K., Hashimoto, H., Sato, N., Kondo, M., et al. (2013). Role of galactolipid biosynthesis in coordinated development of photosynthetic complexes and thylakoid membranes during chloroplast biogenesis in Arabidopsis. Plant J. 73, 250-261. doi: 10.1111/tpj.12028

Kopečná, J., Pilný, J., Krynická, V., Tomčala, A., Kis, M., Gombos, Z., et al. (2015). Lack of phosphatidylglycerol inhibits chlorophyll biosynthesis at multiple sites and limits chlorophyllide reutilization in Synechocystis sp. strain PCC 6803. Plant Physiol. 169, 1307-1317. doi: 10.1104/pp.15.01150

Kubota, H., Sakurai, I., Katayama, K., Mizusawa, N., Ohashi, S., Kobayashi, M., et al. (2010). Purification and characterization of photosystem I complex from Synechocystis sp. PCC 6803 by expressing histidine-tagged subunits. Biochim. Biophys. Acta 1797, 98-105. doi: 10.1016/j.bbabio.2009. 09.001

Kurisu, G., Zhang, H., Smith, J. L., and Cramer, W. A. (2003). Structure of the cytochrome $\mathrm{b}_{6} \mathrm{f}$ complex of oxygenic photosynthesis: tuning the cavity. Science 302, 1009-1014. doi: 10.1126/science. 1090165

Laird, D. M., Parce, J. W., Montgomery, R. I., and Cunningham, C. C. (1986). Effect of phospholipids on the catalytic subunits of the mitochondrial F0.F1-ATPase. J. Biol. Chem. 261, 14851-14856.

Liu, Z., Yan, H., Wang, K., Kuang, T., Zhang, J., Gui, L., et al. (2004). Crystal structure of spinach major light-harvesting complex at $2.72 \AA$ resolution. Nature 428, 287-292. doi: 10.1038/nature02373

Loll, B., Kern, J., Saenger, W., Zouni, A., and Biesiadka, J. (2005). Towards complete cofactor arrangement in the $3.0 \AA$ resolution structure of photosystem II. Nature 438, 1040-1044. doi: 10.1038/nature04224

Loll, B., Kern, J., Saenger, W., Zouni, A., and Biesiadka, J. (2007). Lipids in photosystem II: interactions with protein and cofactors. Biochim. Biophys. Acta 1767, 509-519. doi: 10.1016/j.bbabio.2006.12.009

Mazor, Y., Borovikova, A., and Nelson, N. (2017). The Structure of plant photosystem I super-complex at $2.8 \AA$ resolution. Nat. Plants 3:e07433. doi: 10.7554/eLife.07433

Mazor, Y., Nataf, D., Toporik, H., and Nelson, N. (2014). Crystal structures of virus-like photosystem I complexes from the mesophilic cyanobacterium Synechocystis PCC 6803. Elife 3:e01496. doi: 10.7554/eLife.01496

Melis, A., and Brown, J. S. (1980). Stoichiometry of system I and system II reaction centers and of plastoquinone in different photosynthetic membranes. Proc. Natl. Acad. Sci. U.S.A. 77, 4712-4716. doi: 10.1073/pnas.77.8.4712

Mendiola-Morgenthaler, L., Eichenberger, W., and Boschetti, A. (1985). Isolation of chloroplast envelopes from Chlamydomonas. Lipid and polypeptide composition. Plant Sci. 41, 97-104.

Murakami, A., Kim, S.-J., and Fujita, Y. (1997). Changes in photosystem stoichiometry in response to environmental conditions for cell growth observed with the cyanophyte Synechocystis PCC 6714. Plant Cell Physiol. 38, 392-397. doi: 10.1093/oxfordjournals.pcp.a029181

Murata, N., Sato, N., Omata, T., and Kuwabara, T. (1981). Separation and characterization of thylakoid and cell envelope of the blue-green alga (cyanobacterium) Anacystis nidulans. Plant Cell Physiol. 22, 855-866.

Murphy, D. J. (1982). The importance of non-planar bilayer regions in photosynthetic membranes and their stabilisation by galactolipids. FEBS Lett. 150, 19-26. doi: 10.1016/0014-5793(82)81297-0

Nussberger, S., Dörr, K., Wang, D. N., and Kühlbrandt, W. (1993). Lipid-protein interactions in crystals of plant light-harvesting complex. J. Mol. Biol. 234, 347-356. doi: 10.1006/jmbi.1993.1591

Pan, X., Liu, Z., Li, M., and Chang, W. (2013). Architecture and function of plant light-harvesting complexes II. Curr. Opin. Struct. Biol. 23, 515-525. doi: $10.1016 /$ j.sbi.2013.04.004

Peter, G. F., and Thornber, J. P. (1991). Biochemical composition and organization of higher plant photosystem II light-harvesting pigment-proteins. J. Biol. Chem. 266, 16745-16754.

Qin, X., Suga, M., Kuang, T., and Shen, J.-R. (2015). Structural basis for energy transfer pathways in the plant PSI-LHCI supercomplex. Science 348, 989-995. doi: 10.1126/science.aab0214

Sakurai, I., Hagio, M., Gombos, Z., Tyystjärvi, T., Paakkarinen, V., Aro, E.M., et al. (2003). Requirement of phosphatidylglycerol for maintenance of photosynthetic machinery. Plant Physiol. 133, 1376-1384. doi: 10.1104/pp.103. 026955

Sakurai, I., Mizusawa, N., Ohashi, S., Kobayashi, M., and Wada, H. (2007). Effects of the lack of phosphatidylglycerol on the donor side of photosystem II. Plant Physiol. 144, 1336-1346. doi: 10.1104/pp.107.098731 
Sakurai, I., Shen, J.-R., Leng, J., Ohashi, S., Kobayashi, M., and Wada, H. (2006). Lipids in oxygen-evolving photosystem II complexes of cyanobacteria and higher plants. J. Biochem. 140, 201-209. doi: 10.1093/jb/mvj141

Samson, G., Herbert, S. K., Fork, D. C., and Laudenbach, D. E. (1994). Acclimation of the photosynthetic apparatus to growth irradiance in a mutant strain of Synechococcus lacking iron superoxide dismutase. Plant Physiol. 105, 287-294. doi: $10.1104 /$ pp.105.1.287

Santiago, E., López-Moratalla, N., and Segovia, J. L. (1973). Correlation between losses of mitochondrial ATPase activity and cardiolipin degradation. Biochem. Biophys. Res. Commun. 53, 439-445. doi: 10.1016/0006-291X(73)90 681-5

Sato, N., Tsuzuki, M., Matsuda, Y., Ehara, T., Osafune, T., and Kawaguchi, A. (1995). Isolation and characterization of mutants affected in lipid metabolism of Chlamydomonas reinhardtii. Eur. J. Biochem. 230, 987-993. doi: 10.1111/j. 1432-1033.1995.0987g.x

Schöttler, M. A., and Toth, S. Z. (2014). Photosynthetic complex stoichiometry dynamics in higher plants: environmental acclimation and photosynthetic flux control. Front. Plant Sci. 5:188. doi: 10.3389/fpls.2014.00188

Shipley, G. G., Green, J. P., and Nichols, B. W. (1973). The phase behavior of monogalactosyl, digalactosyl, and sulphoquinovosyl diglycerides. Biochim. Biophys. Acta 311, 531-544. doi: 10.1016/0005-2736(73)90128-4

Standfuss, J., Terwisscha van Scheltinga, A. C., Lamborghini, M., and Kühlbrandt, W. (2005). Mechanisms of photoprotection and nonphotochemical quenching in pea light-harvesting complex at $2.5 \AA$ resolution. EMBO J. 24, 919-928. doi: 10.1038/sj.emboj.7600585

Stroebel, D., Choquet, Y., Popot, J.-L., and Picot, D. (2003). An atypical haem in the cytochrome $b_{6} f$ complex. Nature 426, 413-418. doi: 10.1038/nature02155
Umena, Y., Kawakami, K., Shen, J.-R., and Kamiya, N. (2011). Crystal structure of oxygen-evolving photosystem II at a resolution of $1.9 \AA$ A. Nature 473, 55-60. doi: 10.1038/nature09913

Wei, X., Su, X., Cao, P., Liu, X., Chang, W., Li, M., et al. (2016). Structure of spinach photosystem II-LHCII supercomplex at $3.2 \AA$ resolution. Nature 534, 69-74. doi: $10.1038 /$ nature 18020

Yu, B., and Benning, C. (2003). Anionic lipids are required for chloroplast structure and function in Arabidopsis. Plant J. 36, 762-770. doi: 10.1046/j.1365-313X. 2003.01918.x

Yu, B., Xu, C., and Benning, C. (2002). Arabidopsis disrupted in SQD2 encoding sulfolipid synthase is impaired in phosphate-limited growth. Proc. Natl. Acad. Sci. U.S.A. 99, 5732-5737. doi: 10.1073/pnas.082696499

Yu, S. G., Stefansson, H., Romanowska, E., and Albertsson, P. Å. (1994). Two dimensional electrophoresis of thylakoid membrane proteins and its application to microsequencing. Photosynth. Res. 41, 475-486. doi: 10.1007/ BF02183049

Conflict of Interest Statement: The authors declare that the research was conducted in the absence of any commercial or financial relationships that could be construed as a potential conflict of interest.

Copyright (c) 2017 Kobayashi, Endo and Wada. This is an open-access article distributed under the terms of the Creative Commons Attribution License (CC BY). The use, distribution or reproduction in other forums is permitted, provided the original author(s) or licensor are credited and that the original publication in this journal is cited, in accordance with accepted academic practice. No use, distribution or reproduction is permitted which does not comply with these terms. 